\title{
EFECTOS DE VARIAR LA POSICIÓN ESPACIAL DE LOS ESTÍMULOS EN LA ADQUISICIÓN Y TRANSFERENCIA DE DISCRIMINACIONES CONDICIONALES EN HUMANOS
}

\author{
Enoc Obed De la Sancha Villaํㅜ, Gelacio Guzmán-Díaz ${ }^{1}$, Mario Serrano* \\ ${ }^{1}$ Universidad Veracruzana
}

Recibido, abril 25/2014

Concepto de evaluación, mayo 12/2014

Aceptado, mayo 28/2014
Referencia: De la Sancha Villa, E. O., Guzmán-Díaz, G. \& Serrano, M. (2015). Efectos de variar la posición espacial de los estímulos en la adquisición y transferencia de discriminaciones condicionales en humanos. Acta Colombiana de Psicología, 18(1), 47-54. DOI: 10.14718/ ACP.2015.18.1.5

\section{Resumen}

\begin{abstract}
Se evaluaron los efectos de variar la posición espacial de los estímulos de muestra y de comparación de una tarea de igualación a la muestra sobre la adquisición y transferencia intramodal y extramodal de discriminaciones condicionales por humanos. En el estudio participaron tres grupos de estudiantes universitarios. Entre grupos, las cuatro relaciones de igualación entrenadas se correlacionaron diferencialmente con cuatro posiciones espaciales de los estímulos de comparación (Grupo 1), con cuatro posiciones espaciales de los estímulos de muestra (Grupo 2) o bien con cuatro figuras geométricas (Grupo 3). La ejecución en las pruebas de transferencia intramodal y extramodal fue superior al criterio de ejecución preestablecido experimentalmente para la mayoría de los participantes del Grupo 1. En los otros dos grupos únicamente la mitad de los participantes alcanzó una ejecución similar. Estos resultados concuerdan con la clasificación de los casos paradigmáticos de la función selectora de la propuesta taxonómica de Ribes y López (1985) y sugieren un efecto positivo de la variabilidad en las condiciones de entrenamiento sobre la transferencia de la ejecución.

Palabras clave: Variación espacial, discriminación condicional, transferencia, igualación a la muestra, humanos

\section{EFFECTS OF VARYING THE SPATIAL POSITION OF STIMULI IN THE ACQUISITION AND TRANSFER OF CONDITIONAL DISCRIMINATION IN HUMANS}

\begin{abstract}
The effects of varying the spatial placement of sample and comparison stimuli of a matching-to-sample task were evaluated upon the acquisition and transfer (intramodal and extramodal) of a conditional discrimination by humans. Three groups of college students participated in the experiment. Between groups, four matching-relations were differentially correlated with four spatial placements of the comparison stimuli (Group 1), with four spatial placements of the sample stimuli (Group 2) or with four geometric shapes (Group 3). Performance in both intramodal and extramodal transfer tests was higher than an experimentally predefined criterion for most participants from Group 1. In the remaining groups only half of the participants reached a similar performance. These results agree with the classification of the paradigmatic cases of the selector function from the taxonomic proposal offered by Ribes and López (1985) and suggest a positive effect of variability of training conditions upon the transfer of performance.
\end{abstract}

Key words: Spatial variation, conditional discrimination, transfer, matching-to-sample, humans

\footnotetext{
* Universidad Veracruzana, Centro de Estudios e Investigaciones en Conocimiento y Aprendizaje Humano. Av. Orizaba No. 203. Fraccionamiento Veracruz. Xalapa, Veracruz. México, C. P. 91020 . Teléfono +52 (228) 8903465 ó bien al correo electrónico mserrano@ uv.mx. La redacción final de este trabajo se vio parcialmente apoyada por el proyecto CONACYT No. 180619 a cargo del último autor.
} 


\title{
EFEITOS DE VARIAR A POSIÇÃO ESPACIAL DOS ESTÍMULOS NA AQUISIÇÃO E TRANSFERÊNCIA DE DISCRIMINAÇÓES CONDICIONAIS EM HUMANOS
}

\begin{abstract}
Resumo
Avaliaram-se os efeitos de variar a posição espacial dos estímulos de mostra e de comparação de uma tarefa de igualação à mostra sobre a aquisição e transferência intramodal e extramodal de discriminações condicionais por humanos. No estudo participaram três grupos de estudantes universitários. Entre grupos, a quatro relações de igualação treinadas foram correlacionadas diferencialmente com quatro posições espaciais dos estímulos de comparação (Grupo 1), com quatro posições espaciais dos estímulos de mostra (Grupo 2) ou com quatro figuras geométricas (Grupo 3). A execução nos testes de transferência intramodal e extramodal foi superior ao critério de execução preestabelecido experimentalmente para a maioria dos participantes do Grupo 1. Nos outros dois grupos somente a metade dos participantes atingiu uma execução similar. Estes resultados concordam com a classificação dos casos paradigmáticos da função seletora da proposta taxonômica de Ribes e López (1985) e sugerem um efeito positivo da variabilidade nas condições de treinamento sobre a transferência da execução. Palavras chave: variação espacial, discriminação condicional, transferência, igualação à mostra, humanos
\end{abstract}

\section{INTRODUCCIÓN}

En el contexto de la propuesta taxonómica de Ribes y López (1985), la llamada función selectora describe una interacción psicológica caracterizada porque la funcionalidad de las propiedades fisicoquímicas de los objetos y eventos con los que el organismo interactúa, varía momento a momento. Una misma propiedad fisicoquímica puede tener más de un papel funcional al interior de un arreglo de contingencias, o bien diversas propiedades fisicoquímicas pueden compartir el mismo papel funcional, dependiendo de la presencia/ausencia de un segmento de estímulo anterior o concurrente denominado evento selector.

Las contingencias de ocurrencia (Ribes, 1997) que posibilitan la evolución de la función selectora pueden identificarse en aquellas que están vigentes en las tareas de igualación a la muestra. Como es bien conocido, en estas tareas se presentan un estímulo de muestra y dos o más estímulos de comparación, de entre los cuales un participante debe elegir aquel que guarda con el estímulo de muestra una relación preestablecida. Si el comportamiento del participante tiene o no lugar al nivel de la función selectora, éste se infiere a partir de la ejecución exitosa en pruebas de transferencia en las que la retroalimentación de la ejecución se cancela y se introducen nuevas instancias de estímulo con modalidades pertinentes de igualación también novedosas (véase Varela \& Quintana, 1995).

Dado que la independencia del responder respecto de las propiedades fisicoquímicas de los estímulos que caracteriza a la función selectora se considera una condición crítica para transitar hacia las llamadas funciones sustitutivas de contingencias, en el marco de la propuesta taxonómica de Ribes y López (1985) la mayoría de los estudios que han utilizado tareas de igualación a la muestra se han enfocado a evaluar variables que se relacionan con la evolución de la función selectora sólo tangencialmente (véase Moreno, Cepeda, Tena, Hickman \& Plancarte, 2005; Ribes, 1998; Tena, Hickman, Moreno, Cepeda \& Larios, 2001). En general, en tales estudios las preguntas de investigación se han enfocado a evaluar los efectos de la agregación de variables lingüísticas más que a la manipulación de parámetros tales como la geografía o la morfología de los estímulos, considerados por los autores de la taxonomía de referencia como los primeros a ser tomados en cuenta en el caso de la función selectora.

Al margen del señalamiento por parte de Ribes y López (1985), la manipulación de parámetros morfológicos y geográficos parece importante, al menos por una razón simple, pero importante. Específicamente, al igual que en el caso de las llamadas funciones contextual y suplementaria, la configuración del comportamiento al nivel de la función selectora constituye un proceso psicológico regulado intrasituacionalmente, es decir, que depende de las propiedades fisicoquímicas de objetos y eventos presentes en una situación interactiva. El peso funcional de tales propiedades, objetos y eventos, sin embargo, no es necesariamente homogéneo. En interacción con el tipo de contingencias de ocurrencia implicadas, una misma variable puede tener efectos diferentes dependiendo del segmento de estímulo o de respuesta sobre el que se aplica. En el caso de las tareas de igualación a la muestra, por ejemplo, se ha observado que en el caso de las palomas imponer requisitos de respuesta a los estímulos de muestra mejora la precisión de la ejecución (e.g., Eckerman, Lanson \& Cumming, 1968; Roberts, 1972), mientras que imponerlos sobre los estímulos de comparación tiene el 
efecto inverso (e.g., Lydersen, Perkins \& Chairez, 1977; Wilkie \& Spetch, 1978).

Entre los numerosos estudios que utilizan tareas de igualación a la muestra realizados en el contexto de la propuesta taxonómica de Ribes y López (1985), sólo un experimento conducido por Carpio, Pacheco, García y Sierra (1991) puede identificarse como un estudio enfocado al análisis de las variables propias de la función selectora. Específicamente, dichos autores evaluaron los efectos de variar la posición de los estímulos de comparación de una tarea de igualación a la muestra de primer orden, sobre la adquisición y transferencia intramodal y extramodal de una discriminación condicional basada en la relación de identidad. En general, dichos autores observaron que tanto para el grupo expuesto a diferentes configuraciones posicionales de los estímulos de comparación, como para aquel expuesto a una sola configuración posicional, la ejecución en ambas pruebas de transferencia fue prácticamente equivalente.

La ausencia de diferencias entre grupos en el experimento de Carpio et al. (1991) se atribuyó a que en ningún caso la posición de los estímulos de comparación "constituyó un factor contingencialmente vinculado con la efectividad de la ejecución" (p. 51). En este sentido, en el presente estudio se compararon los efectos de variar la posición de los estímulos de muestra y de los estímulos de comparación de una tarea de igualación a la muestra, correlacionando diferencialmente tales posiciones con cada una de las relaciones de igualación entrenadas. En la medida que correlaciones por el estilo permiten suponer que una tarea de igualación a la muestra de primer orden se ha tornado en una tarea de segundo orden, para un tercer grupo de participantes las posiciones de los estímulos de muestra y de comparación permanecieron constantes y las relaciones de igualación se correlacionaron con un estímulo selector discriminante (Serrano, García \& López, 2007). Es de destacar que una situación por el estilo adicionalmente permitiría explorar si los posibles efectos de la correlación entre la posición de los estímulos y las relaciones de igualación son similares en el caso de variables morfológicas.

\section{MÉTODO}

\section{Participantes}

En el estudio participaron 12 estudiantes de primer semestre de la Facultad de Psicología de la Universidad Veracruzana (UV), Campus Xalapa. Los participantes fueron siete mujeres y cinco hombres entre los 18 y 20 años de edad, quienes reportaron no tener experiencia en tareas de igualación a la muestra o algún otro procedimiento experimental.

\section{Aparatos}

El estudio se llevó a cabo en los cubículos de experimentación del Laboratorio de Aprendizaje Humano Sidney Bijou del Centro de Estudios e Investigaciones en Conocimiento y Aprendizaje Humano de la UV. Además de repisa y silla, cada cubículo contó con una computadora personal estándar (HP Compaq Modelo, dc5850) provista con monitor, teclado y mouse. Las instrucciones e instancias de estímulo de las tareas experimentales fueron elaboradas en mapas de bites independientes y organizadas mediante el programa SuperLab Pro ${ }^{\circledR}$ (Versión 2.0) en un ambiente Windows ${ }^{\circledR}$. El mouse funcionó como dispositivo para las respuestas de igualación, las cuales fueron registradas automáticamente por las computadoras. Las sesiones experimentales se realizaron entre las 14:00 y las 16:00 horas.

\section{Diseño}

Los participantes fueron divididos de forma aleatoria en tres grupos de cuatro participantes cada uno (Grupo 1, Grupo 2 y Grupo 3). Cada grupo de participantes fue expuesto a una preprueba, tres sesiones de entrenamiento, una prueba de transferencia intramodal y una prueba de transferencia extramodal. Para los grupos 1 y 2 se utilizó una tarea de

Tabla 1.

\begin{tabular}{ccccccc}
\hline Grupo & Tipo de tarea & Variación espacial & Fase 1 & Fase 1 & Fase 1 & Fase 1 \\
\hline 1 & Primer orden & Estímulos de comparación & & & & \\
2 & Primer orden & Estímulos de muestra & Preprueba & Entrenamiento & $\begin{array}{c}\text { Transferencia } \\
\text { Intramodal }\end{array}$ & $\begin{array}{c}\text { Transferencia } \\
\text { Extramodal }\end{array}$ \\
3 & Segundo orden & N/A & & & & \\
\hline & & SESIONES & 1 & 3 & 1 & 1 \\
\hline
\end{tabular}


igualación a la muestra de primer orden, mientras para el grupo 3 se utilizó una tarea de igualación a la muestra de segundo orden con un estímulo selector discriminante (Serrano, García \& López, 2007). La tabla 1 describe el diseño del presente experimento.

Para los participantes del grupo 1 el estímulo de muestra se ubicó en el centro de la pantalla mientras una alineación de cuatro estímulos de comparación se presentó en una de cuatro posiciones posibles. Cada posición de la alineación de los estímulos de comparación se correlacionó con una relación de igualación específica: abajo-identidad, arriba-diferencia, derecha-semejanza en forma e izquierdasemejanza en color. Para los participantes del grupo 2, los cuatro estímulos de comparación se presentaron alineados horizontalmente en el centro de la pantalla mientras el estímulo de muestra se ubicó en una de las cuatro esquinas. De manera análoga que para el grupo 1, para el grupo 2 las posiciones del estímulo de muestra se correlacionaron diferencialmente con las relaciones de igualación entrenadas: arriba a la derecha-diferencia, arriba a la izquierdaidentidad, abajo a la derecha-semejanza en forma y abajo a la izquierda-semejanza en color. Para los participantes del grupo 3, el estímulo de muestra se presentó en el centro de la pantalla, los estímulos de comparación se presentaron alineados horizontalmente en la parte inferior y el estímulo selector discriminante se presentó en la parte superior de la pantalla, paralelamente al estímulo de muestra. La figura 1 representa esquemáticamente las características de las tareas de igualación a la muestra de primer y segundo órdenes utilizadas en el presente estudio.

\section{Procedimiento}

1. Preprueba. Para todos los grupos de participantes la preprueba estuvo conformada por 28 ensayos de igualación a la muestra. Los arreglos estuvieron diseñados de tal forma que siempre existió un estímulo de comparación idéntico, uno semejante en color, uno semejante en forma y uno diferente respecto del estímulo de muestra. En todos los casos, los ensayos de la preprueba se extrajeron de los ensayos que se presentarían en las sesiones de entrenamiento. Para los participantes del grupo 1 las instrucciones de la preprueba fueron:

En las siguientes pantallas aparecerán cinco figuras geométricas: una en el centro de la pantalla y cuatro más arriba, abajo, a la derecha o a la izquierda. De estas últimas elige la figura que corresponde con la del centro, de acuerdo con lo que indican sus posiciones. Para registrar tu respuesta, ubica el puntero del "mouse" dentro de la figura que elegiste y oprime el botón izquierdo. Para comenzar, oprime el botón "COMENZAR".

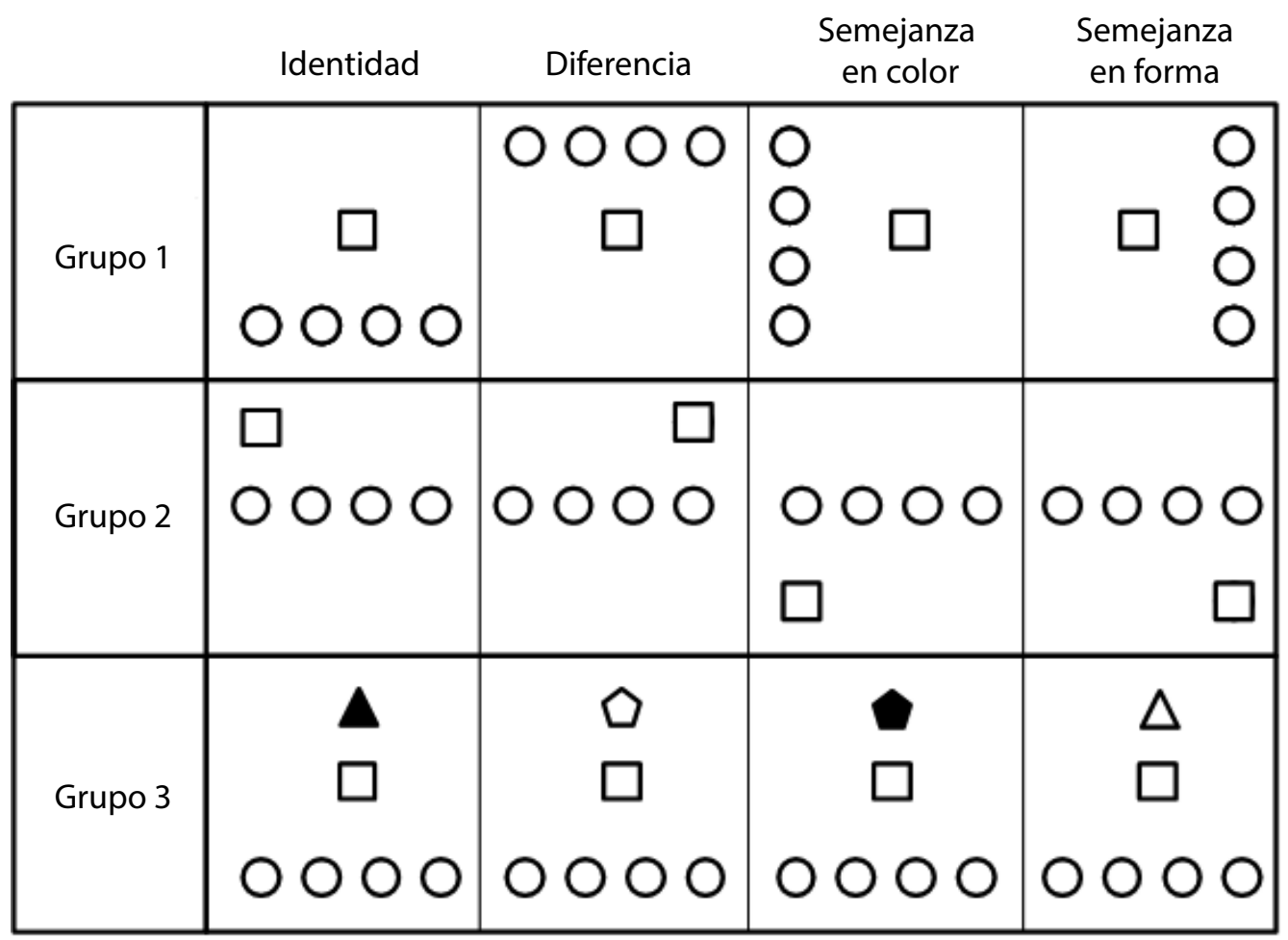

Figura 1. Características de las tareas de igualación a la muestra. 
Para los participantes del grupo 2 las instrucciones de la preprueba fueron:

En las siguientes pantallas aparecerán cinco figuras geométricas: cuatro en el centro de la pantalla y una más en cualquiera de las cuatro esquinas. Elige la figura del centro que corresponde con la figura en alguna de las esquinas, de acuerdo con lo que indica su posición. Para registrar tu respuesta, ubica el puntero del "mouse" dentro de la figura que elegiste y oprime el botón izquierdo. Para comenzar, oprime el botón "COMENZAR".

Para los participantes del grupo 3 las instrucciones de la preprueba fueron:

En las siguientes pantallas aparecerán seis figuras geométricas: una en la parte superior de la pantalla, una en el centro y cuatro más en la parte inferior. Elige la figura de abajo que corresponde con la del centro, de acuerdo con lo que indica la figura de arriba. Para registrar tu respuesta, ubica el puntero del "mouse" dentro de la figura que elegiste y oprime el botón izquierdo. Para comenzar, oprime el botón "COMENZAR".

2. Entrenamiento. Posterior a la preprueba se realizaron tres sesiones de entrenamiento. Cada sesión de entrenamiento consistió en una tarea de igualación a la muestra similar a la implementada en la preprueba para cada grupo, excepto por tres aspectos. En primer lugar, se agregó a las instrucciones iniciales el enunciado Te informaremos si tu respuesta fue correcta o incorrecta. En segundo lugar, se programaron 42 ensayos por cada relación de igualación, los cuales se presentaron en cuatro bloques: identidad, semejanza en forma, semejanza en color y diferencia, en ese orden. Así pues, cada sesión de entrenamiento consistió en 168 ensayos de igualación. En tercer lugar, las respuestas de igualación, acertadas y erróneas, produjeron, respectivamente, la presentación de las palabras correcto e incorrecto durante $5 \mathrm{~s}$ en la pantalla de la computadora.

3. Pruebas de transferencia. Inmediatamente después de la tercera sesión de entrenamiento, los tres grupos de participantes fueron expuestos a pruebas de transferencia intramodal y extramodal, en ese orden. El aviso que señaló (durante $10 \mathrm{~s}$ ) el inicio de las pruebas de transferencia fue: En las siguientes pantallas ya no se te informará si tu respuesta fue correcta o incorrecta. La prueba de transferencia intramodal implicó la presentación de instancias de estímulo con nuevos colores y figuras como estímulos de muestra y de comparación. En el caso del grupo 3, las figuras geométricas que fungieron como estímulos selectores discriminantes fueron las mismas que en las sesiones de entrenamiento. En la prueba de transferencia extramodal la modalidad pertinente de igualación cambió del color al contorno de las figuras. Los estímulos de muestra y de comparación fueron similares a los utilizados en la prueba de transferencia intramodal, excepto porque se presentaron en color blanco y sus contornos variaron. En el caso del grupo 3, nuevamente se presentaron las figuras geométricas que fungieron como estímulos selectores discriminantes durante el entrenamiento.

Cada prueba de transferencia estuvo conformada por 56 ensayos de igualación a la muestra, los cuales se distribuyeron en cuatro bloques de 14 ensayos. En cada bloque estuvo vigente una de las cuatro relaciones de igualación entrenadas, las cuales se presentaron de manera similar a las sesiones de entrenamiento: identidad, semejanza en forma, semejanza en color (o contorno) y diferencia. En la prueba de transferencia intramodal los arreglos estuvieron diseñados de tal forma que siempre existió un estímulo de comparación idéntico, uno semejante en color, uno semejante en forma y otro diferente. En la prueba de transferencia extramodal los arreglos estuvieron diseñados de tal forma que siempre existió un estímulo de comparación idéntico, uno semejante en contorno, uno semejante en forma y otro diferente.

\section{RESULTADOS}

En el presente estudio la precisión de la ejecución se estimó con base en el porcentaje de ensayos en los que los participantes eligieron el estímulo comparativo predeterminado experimentalmente según la posición de los estímulos comparativos (Grupo 1), los estímulos de muestra (Grupo 2), o bien los estímulos selectores discriminantes presentados (Grupo 3). En cada sesión, el número de ensayos en los que se eligió el estímulo comparativo predeterminado experimentalmente se dividió entre el total de ensayos programados en esa sesión y/o fase del experimento y el resultado se multiplicó por 100. Los porcentajes resultantes se despliegan en la figura 2 como barras blancas para la preprueba (PP), círculos negros para las sesiones de entrenamiento (S1, S2 y S3), barras blancas con líneas diagonales para la prueba de transferencia intramodal (TIM) y barras negras para la prueba de transferencia extramodal (TEM).

La figura 2 muestra el porcentaje de ensayos en los que se eligió el estímulo comparativo predeterminado experimentalmente para los participantes de cada grupo a lo largo del experimento. En la figura se observa que para la mayoría de los participantes tales porcentajes fueron inferiores al $30 \%$ en la preprueba. La única excepción fue P12 del grupo 3, para el que se registró un 33\% de ensayos consistentes con lo predeterminado experimentalmente. En el entrenamiento, para la mayoría de los participantes se observa un incremento gradual en los porcentajes 
calculados a lo largo de las tres sesiones de entrenamiento implementadas. Sin embargo, se observa que mientras para los participantes del grupo 2 la ejecución siempre fue igual o superior al $80 \%$, para dos participantes del grupo $1 \mathrm{y}$ tres participantes del grupo 3 los porcentajes en la primera sesión de entrenamiento fueron inferiores al $80 \%$. Con excepción de P1 del grupo 1, la ejecución terminal en el entrenamiento fue cercana o superior al $90 \%$ de ensayos consistentes con lo predeterminado experimentalmente para todos los participantes.

La precisión de la ejecución en ambas pruebas de transferencia superó el $70 \%$ de ensayos consistentes con lo predeterminado experimentalmente para tres participantes del grupo 1 (P2, P3 y P4), dos participantes del grupo 2 (P7 y P8) y dos participantes del grupo 3 (P9 y P12). Para el participante restante del grupo 1 (P1) la ejecución fue cercana al $25 \%$ de ensayos consistentes con lo predeterminado experimentalmente en ambas pruebas. Para P5 y P6 del grupo 2, en la prueba de transferencia intramodal se registraron ejecuciones de 100 y $69 \%$ de ensayos consistentes con lo predeterminado, respectivamente, mientras en la prueba de transferencia extramodal las ejecuciones fueron de 64 y $44 \%$ de ensayos consistentes con lo experimentalmente predeterminado. En el caso del grupo 3, mientras para P10 la ejecución fue cercana al $25 \%$ en ambas pruebas, para P11 se registraron ejecuciones de 67 y 53\% de ensayos consistentes con lo predeterminado por el experimentador en las pruebas de transferencia intramodal y extramodal, respectivamente.

\section{GRUPO 1}
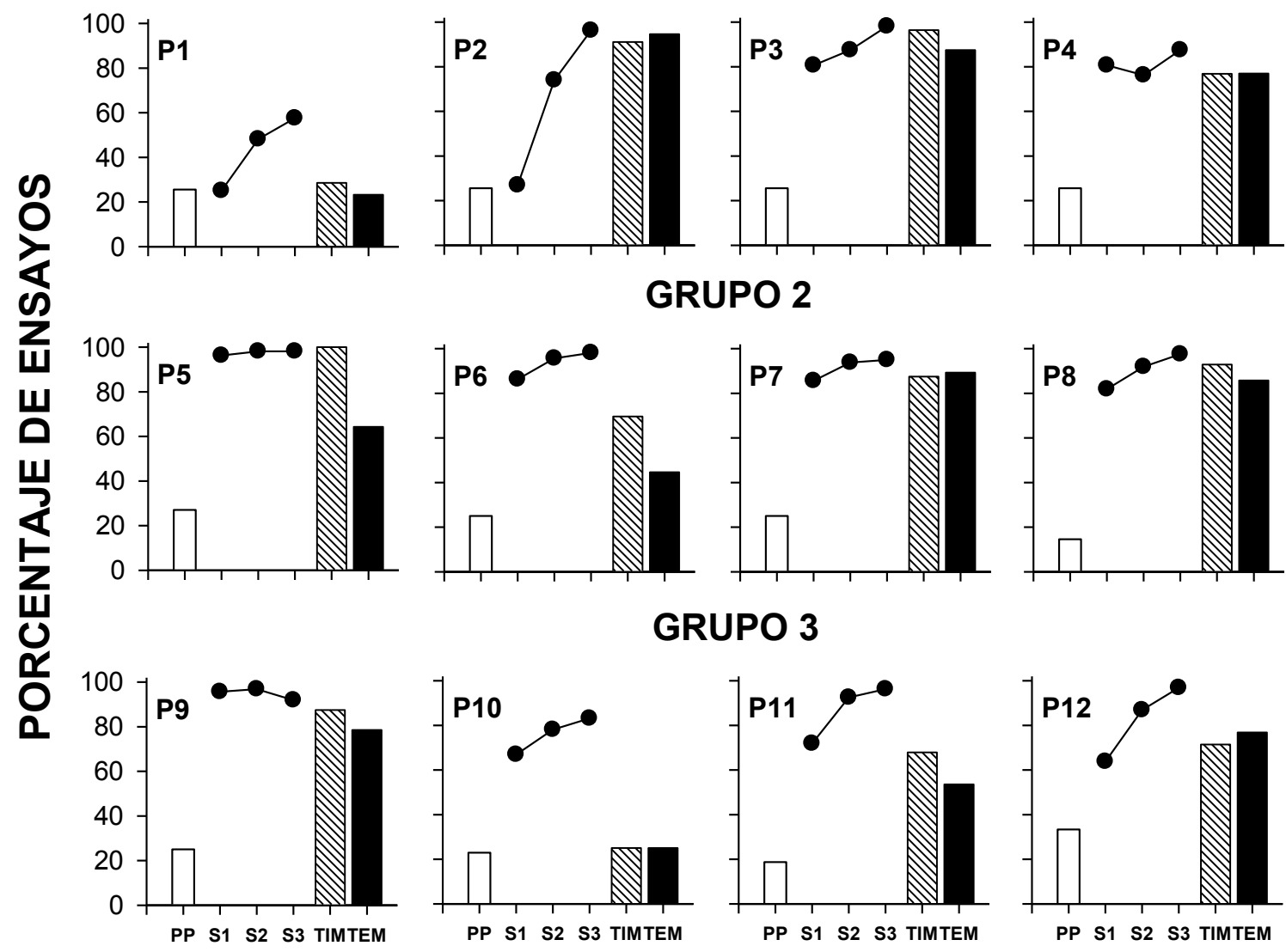

GRUPO 2
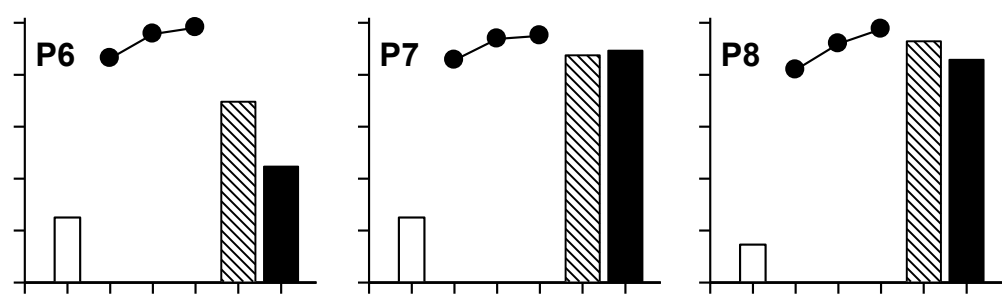

GRUPO 3
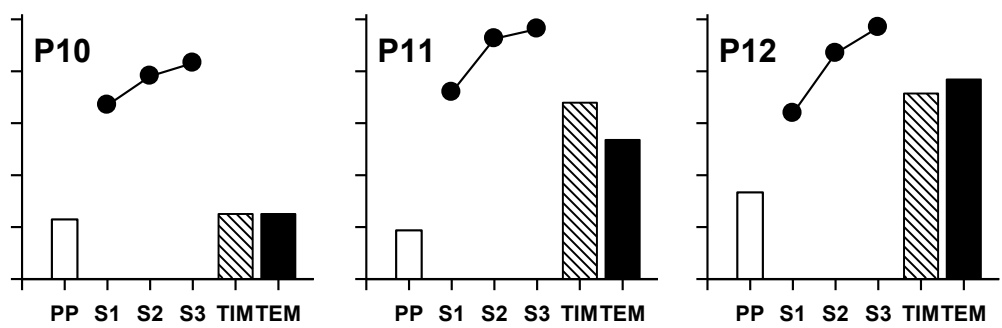

Figura 2. Porcentaje de ensayos en los que se eligió el estímulo comparativo predeterminado experimentalmente para cada participante a lo largo del estudio $[\mathrm{PP}=$ Preprueba, $\mathrm{S}=$ Sesión, TIM = Transferencia intramodal, TEM = Transferencia extramodal]. 


\section{DISCUSIÓN}

El presente estudio comparó los efectos de correlacionar las relaciones de igualación entrenadas con la posición espacial de los estímulos de muestra, la posición espacial de los estímulos de comparación o bien con un tercer segmento de estímulo de una tarea de igualación a la muestra, sobre la adquisición y transferencia de una discriminación condicional en humanos. La configuración del comportamiento al nivel de la función selectora reconocida en la propuesta taxonómica de Ribes y López (1985), estimada a partir de una ejecución igual o superior al $70 \%$ de ensayos consistentes con lo predeterminado experimentalmente al cancelar la retroalimentación e introducir variaciones instanciales y modales, se observó para la mayoría de los participantes del Grupo 1, es decir, aquellos para los que se variaron espacialmente los estímulos de comparación. En los dos grupos restantes, en los que la posición de los estímulos de muestra y diferentes segmentos de estímulo adicionales se correlacionaron con las relaciones de igualación entrenadas, respectivamente, sólo la mitad de los participantes superó dicho porcentaje en ambas pruebas de transferencia. De acuerdo con lo señalado en la sección introductoria, los resultados del presente estudio muestran que el peso funcional de las variaciones geográficas de los estímulos depende del segmento sobre el que se aplican, es decir, suplementario o selector, al tiempo que sugieren cierto grado de equivalencia funcional entre las variables espaciales en el caso del segundo de tales segmentos y las variables morfológicas.

Los resultados del presente estudio concuerdan con los previamente descritos por Carpio et al. (1991), en el sentido de que cuando la posición de los estímulos está vinculada contingencialmente con la precisión del responder, se observan ejecuciones diferenciales dependiendo del tipo funcional de estímulo cuya posición se manipula. Al respecto, llama la atención que en el experimento que aquí se reporta no haya sido la variación espacial del estímulo de muestra, es decir el potencial mediador de la interacción selectora, aquella que produjo el mayor número de ejecuciones superiores al $70 \%$ de ensayos consistentes con lo predeterminado experimentalmente en las pruebas de transferencia intramodal y extramodal. Como se señaló anteriormente, estudios previos con palomas encontraron que imponer requisitos de respuesta a los estímulos de muestra mejoró la precisión de la ejecución (e.g., Eckerman et al., 1968; Roberts, 1972), mientras que imponer tales requisitos sobre los estímulos de comparación tuvo el efecto inverso (e.g., Lydersen et al., 1977; Wilkie \& Spetch, 1978). Dado que de acuerdo con Ribes y López (1985) el comportamiento al nivel de la función selectora puede tener lugar tanto en animales como en humanos, los resultados de los estudios recién aludidos permitían adelantar que la variación espacial de los estímulos de muestra produciría mejores ejecuciones que la variación espacial de los estímulos de comparación.

Esta diferencia con lo esperado puede interpretarse de al menos dos maneras. Por un lado, que la función selectora se haya establecido en un mayor número de participantes cuando se varió la posición de los estímulos de comparación que cuando se varió la posición de los estímulos de muestra, confirmaría que mientras la covariación entre propiedades fisicoquímicas de los eventos selectores y los eventos suplementarios justifica y constituye un caso funcional de cierta complejidad (i.e., la condicionalidad del evento suplementario), la covariación entre diferentes propiedades de los eventos selectores (i.e., fisicoquímicas y geográficas) no justifica un caso funcional adicional y, más bien, debe interpretarse como una concreción particular de alguno de los otros tres casos de la función selectora descritos por Ribes y López (1985). Al respecto, el hecho de que la velocidad de adquisición de la discriminación condicional haya sido mayor para los participantes de los grupos 2 y 3 que para los participantes del grupo 1, no sólo apoya la idea de que el caso funcional en cuestión debe implicar una menor complejidad que el anteriormente señalado, sino adicionalmente que la función selectora puede tener lugar en tal nivel de complejidad intrafunción (i.e., la condicionalidad de la relación suplementaria) a pesar de que el evento selector esté auspiciado por una relación entre las propiedades fisicoquímicas de dos segmentos de estímulo, es decir, tal como en el caso funcional más complejo de la función selectora (i.e., la doble condicionalidad de la relación suplementaria).

En segundo lugar, en la medida que el grado de variabilidad en el entrenamiento de discriminaciones condicionales ha demostrado ser una variable poderosa en la transferencia de la ejecución (e.g., Cepeda, Moreno \& Larios, 2000; Irigoyen, Carpio, Jiménez, Silva, Acuña \& Arroyo, 2002), una posible explicación en torno de las diferencias entre variar la posición espacial de los estímulos de muestra y la de los estímulos de comparación, radica en el hecho de que en este último caso el número de elementos implicados en la variación fue mayor que en el primero (i.e., cuatro versus uno). Además de simple, esta explicación concuerda con la ejecución observada en el caso de los participantes del grupo 3, para los que el número de elementos que variaron entre los bloques de ensayos fue el mismo que para los participantes del grupo 2.

Antes de concluir, debe destacarse que las dos interpretaciones anteriores no son de ninguna manera excluyentes. 
Sin embargo, nuevos estudios deberán abordar experimentalmente ambos planteamientos a fin de confirmar su compatibilidad y, en esa medida, abonar en favor de la correspondencia empírica de la propuesta taxonómica motivo del presente estudio. En este sentido, es deseable que tales estudios tomen en consideración la inclusión de alguna estrategia que permita registrar concurrentemente respuestas compatibles e incompatibles con las respuestas requeridas por la tarea experimental (e.g., Rodríguez-Pérez, Castellanos-Meza \& Díaz-Gámez, 2008).

\section{REFERENCIAS}

Carpio, C. Pacheco, V., García, R., \& Sierra, R. (1991). Efectos del entrenamiento configuracional en tareas de discriminación condicional simple. Revista Mexicana de Análisis de la Conducta, 17, 37-52.

Cepeda, M., Moreno, D. \& Larios, R. (2000). Relación de un entrenamiento variado con opciones textuales y la transferencia en una tarea de discriminación condicional. Revista Psicología y Ciencia Social, 2, 3-16.

Eckerman, D. A., Lanson, R. N., \& Cumming, W. W. (1968). Acquisition and maintenance of matching without a required observing response. Journal of the Experimental Analysis of Behavior, 11, 435-441.

Irigoyen, J. J., Carpio, C., Jiménez, M., Silva, H., Acuña, K., \& Arroyo, A. (2002). Variabilidad en el entrenamiento con retroalimentación parcial en la adquisición de desempeños efectivos. Enseñanza e Investigación en Psicología, 7, 221234.

Lydersen, T., Perkins, D. \& Chairez, H. (1977). Effects of fixedratio sample and choice response requirement upon oddity matching. Journal of the Experimental Analysis of Behavior, 25, 97-101.

Moreno, D., Cepeda, M., Tena, O., Hickman, H., \& Plancarte, P. (2005). Conducta gobernada por reglas: Implicaciones educativas. En C. Carpio \& J. J. Irigoyen (Eds.), Psicología y educación: Aportaciones desde la teoría de la conducta (pp.175-212). México: Universidad Nacional Autónoma de México-Facultad de Estudios Superiores Iztacala.

Ribes, E. (1997). Causality and contingency: Some conceptual considerations. The Psychological Record, 47, 619-639.

Ribes, E. (1998). La investigación básica concebida como programa científico. En V. Alcaraz \& A. Bouzas (Eds.), Las aportaciones mexicanas a la psicología. La perspectiva de la investigación (pp. 89-101). México: UNAM.

Ribes, E., \& López, F. (1985). Teoría de la conducta: Un análisis de campo y paramétrico. México: Trillas.

Roberts, W. A. (1972). Short-term memory in the pigeon: Effects of repetition and spacing. Journal of Experimental Psychology, 94, 74-83.

Rodríguez-Pérez, M. E., Castellanos-Meza, F. A., \& Díaz-Gámez, L. L. (2008). Análisis del discurso didáctico usando una tarea de igualación a la muestra de segundo orden. Acta Colombiana de Psicología, 11, 65-74.

Serrano, M., García, G., \& López, A. (2007). Estímulos selectores instruccionales y discriminantes en igualación a la muestra con humanos. Alternativas en Psicología, 12, 91102.

Tena, O., Hickman, H., Moreno, D., Cepeda, M., \& Larios, R. (2001). Estudios sobre comportamiento complejo. En G. Mares \& Y. Guevara (Eds.), Psicología interconductual: Avances en la investigación básica (pp. 59-110). México: Universidad Nacional Autónoma de México-Facultad de Estudios Superiores Iztacala.

Varela, J., \& Quintana, C. (1995). Comportamiento inteligente y su transferencia. Revista Mexicana de Análisis de la Conducta, 21, 47-66.

Wilkie, D. M., \& Spetch, M. L. (1978). The effect of sample and comparison ratio schedules on delayed matching to sample in the pigeon. Animal Learning \& Behavior, 6, 273-278. 